\title{
IMPLICATIONS OF ZAMBIAN ADOLESCENTS' KNOWLEDGE ABOUT HIV AND AIDS FOR ADHERENCE TO TREATMENT AND PERCEIVED STIGMA
}

\author{
J. Andren ${ }^{1}$, A. Menon ${ }^{2}$, A. McPherson ${ }^{3}$, M. Ngoma ${ }^{4}$, R. Nair ${ }^{5}$, P. Garrud ${ }^{6}$, S. Degun ${ }^{1}$, C. Glazebrook ${ }^{1}$ \\ ${ }^{1}$ Psychiatry, University of Nottingham, Nottingham, UK, ${ }^{2}$ Psychology, Unniversity of Zambia, Lusaka, \\ Zambia, ${ }^{3}$ Bloorview Research Institute, Holland Bloorview Kids Rehabilitation Hospital, Toronto, ON, \\ Canada, ${ }^{4}$ Child Health, University Teaching Hospital, Lusaka, Zambia, ${ }^{5}$ Institute of Work Health and \\ Organisations, University of Nottingham, Nottingham, ${ }^{6}$ School of Graduate Entry Medicine and Health, \\ University of Nottingham, Derby, $U K$
}

Background and aims: A previous randomised trial found that a leaflet designed to promote selfmanagement skills in HIV and AIDS was effective in increasing knowledge and reducing HIV related stigma in a school population of Zambian adolescents This study aims to examine the effectiveness of the leaflet in a clinic population of adolescents with HIV and AIDS and to explore the impact of knowledge on adherence.

Methods: 77 participants were recruited from in the Lusaka region and randomised to receive the HIV leaflet or a control leaflet. Participants completed an HIV knowledge questionnaire, an adapted version of the Medication Adherence Self Report Inventory, a measure of health locus of control (HLC) and a stigma scale at time 1 and at 5 weeks follow-up.

Results: There were no differences in knowledge, HLC, stigma or adherence between the groups at time 1 or at 5 weeks follow-up. In the sample as a whole knowledge was low. 15 young people admitted to missing at least one dose of medication in the previous day and those children had significantly lower knowledge $(\mathrm{p}=0.02)$. Lower knowledge was strongly associated with higher levels of stigma $(\mathrm{r}=-.639, \mathrm{p}<0.001)$ and a more internal HLC $(r=0.575, p<0.001)$. Regression analysis found that knowledge accounted for $28 \%$ of variance in stigma scores after controlling for age and gender.

Conclusion: Children with HIV had low levels of knowledge about HIV and AIDS and a self management leaflet was not effective in increasing knowledge, suggesting a need for more intensive intervention. 